\title{
Prostatakarzinom: Abwarten bei geringem Risiko akzeptabel
}

\section{Eine abwartende Strategie beim lokalen Prostatakarzinom wird immer wieder kontrovers diskutiert. Eine populationsbasierte Kohortenstudie aus Schweden gibt dieser Thematik nun neue Nahrung.}

$\mathrm{n}$ einer schwedischen Studie wurden Langzeitüberlebensdaten von Patienten einbezogen, die zwischen 1997 und 2002 im Alter von bis zu 70 Jahren die Diagnose eins lokalen T1- bis T2Prostatakarzinoms erhalten hatten. Dabei werteten die Autoren als prognostisch relevante Marker auch GleasonScore und PSA-Serumspiegel aus. Indem sie das Patientenregister mit dem schwedischen Sterberegister verknüpften, errechneten die Autoren die Mortalität in dieser Kohorte. Von den 6.849 Patienten wurden 2.021 zunächst nur aktiv überwacht, 3.399 radikal prostatektomiert und 1.429 unterzogen sich einer Strahlentherapie. 2.686 der Patienten hatten ein Prostatakarzinom niedrigen Risikos (T1, Gleason-Score 2-6, SerumPSA-Wert < 10 ng/ml). Bei 1.085 dieser Patienten wurde eine abwartende Strategie mit regelmäßiger Überwachung verfolgt, die übrigen 1.601 Patienten wurden mit kurativer Absicht behandelt. Die Nachbeobachtungszeit betrug bis zu 8,2 Jahre.
Über alle Risikogruppen hinweg ergab sich eine kumulative Prostatakarzinomspezifische Mortalität über zehn Jahre von 3,6\% bei einer abwartenden Strategie und 2,7\% bei Behandlung (Prostatektomie und Bestrahlung zusammengenommen). Werteten die Untersucher nur die Daten der Patienten mit Prostatakarzinomen niedrigen Risikos aus, lag die krankheitsspezifische Mortalität in zehn Jahren bei 2,4\% in der Watchful-Waiting-Gruppe und bei $0,7 \%$ in der Therapiegruppe.

Die Sterberate aus andern Gründen war in der Gesamtkohorte niedriger als erwartet. Das werten die Autoren als Beleg für die Selektion recht gesunder Männer durch den PSA-Test, von denen dann einige die Diagnose eines lokalen Prostatakarzinoms erhalten. Dass das Sterberisiko aus anderen Gründen in der Gruppe mit abwartender Strategie mit 19,2\% höher war als in der Therapiegruppe mit $10,2 \%$, interpretieren die Autoren dagegen mit einer eher abwartenden Haltung bei Männern mit Begleiterkrankungen
- Patienten mit geringerer Lebenserwartung wurden öfter nur überwacht und nicht behandelt.

Fazit: Die Daten aus der populationsbasierten Kohortenstudie zeigen einen gewissen Vorteil der kurativen Therapie gegenüber dem abwartenden Verhalten schon beim Prostatakarzinom mit niedrigem Risiko. Dennoch sehen die Autoren in diesen Daten eine Bestätigung für die abwartende Haltung speziell in dieser Situation, da die prostatakrebsspezifische Sterblichkeit auch bei Verzicht auf sofortige Therapie in zehn Jahren nur bei 2,4\% liegt.

fk

Stattin P et al. Outcomes in localized prostate cancer: National Prostate Cancer Register of Sweden follow-up study. J Natl Cancer Inst 2010; 102: 950-8 